\title{
Cooperative Control with Adaptive Graph Laplacians for Spacecraft Formation Flying
}

\author{
Insu Chang, Soon-Jo Chung, and Lars Blackmore
}

\begin{abstract}
This paper investigates exact nonlinear dynamics and cooperative control for spacecraft formation flying with Earth oblateness ( $J_{2}$ perturbation) and atmospheric drag effects. The nonlinear dynamics for chief and deputy motions are derived by using Gauss' variational equation and the EulerLagrangian formulation, respectively. The proposed cooperative control employs adaptive time-varying Laplacian gains. The tracking and diffusive coupling gains are adapted by the synchronization/tracking errors and distance-based connectivity, thereby defining a time-varying network topology. Moreover, the proposed method relaxes the network structure requirement and permits an unbalanced graph. Nonlinear stability is proven by contraction analysis and incremental input-to-state stability. Numerical examples show the effectiveness of the proposed method.
\end{abstract}

\section{INTRODUCTION}

Spacecraft formation flying has been extensively studied due to its potential applications to many future space science missions such as stellar interferometry. [1]. For spacecraft formation flying, control and modeling of the relative states between the chief (or reference) spacecraft and a deputy spacecraft are core research areas. A number of linearized relative dynamic models exist in the literature. The most popular relative dynamic model is the Hill-Clohessy-Wiltshire (HCW) equation [2], which assumes a circular reference orbit. Tschauner and Hempel derived a new relative dynamic model with elliptic reference orbits [3]. Later, Schweighart and Sedwick [4] and Ross [5] suggested linearized relative dynamics including the $J_{2}$ perturbation for formation flying. A new description of the relative dynamics by the state transition matrix was derived by Gim and Alfriend [6]. If initial states of the relative dynamics are known, we can find the states at any given time. However, the state transition matrix has a somewhat complex and cumbersome form. Unfortunately, all of the models mentioned so far are approximations based on linearization, and so have significant errors away from the linearization point. With relative dynamics, this means that linearized models can typically not be used if the spacecraft are separated by more than a kilometer. This motivates the use of exact, nonlinear models.

A nonlinear model for the relative dynamics with the $J_{2}$ perturbation and atmospheric drag was presented by

I. Chang and S.-J. Chung are with the Department of Aerospace Engineering, University of Illinois at Urbana-Champaign, Urbana, IL 61801, USA. Email: (chang162, sjchung)@illinois.edu.

L. Blackmore is with Jet Propulsion Laboratory, California Institute of Technology, Pasadena, CA 91109, USA. Email: james.c.blackmore@jpl.nasa.gov.
Kechichian [7]. However, some components of the $J_{2}$ perturbation were not considered. A new exact nonlinear reference model and a relative orbital dynamic model with the $J_{2}$ perturbation were suggested by Xu and Wang [8]. However, since the model uses hybrid states (position/velocity and some orbital elements) to describe the dynamics, to use the model in practice requires additional calculation. Moreover, orbital elements are more intuitive than position and velocity to determine orbit shapes. In the present paper, we extend this work by providing exact nonlinear dynamics for reference and relative motion with the $J_{2}$ perturbation and atmospheric drag, and without the need for hybrid states. We derive these dynamics using Gauss' variational equation and the EulerLagrangian formulation.

In addition to spacecraft formation flying, there have been a variety of studies of cooperative control in robots and UAVs [9], [10]. However, unlike the studies in other fields, the dynamics of spacecraft are very complicated and highly nonlinear so that more sophisticated control methods are needed. A more detailed review of spacecraft formation flying guidance and control can be found in [11], [12]. In particular, various architectures for spacecraft formation flying were introduced in [13]. A leader-follower system has been most popular [14], various types of the decentralized coordinate-based control also have been extensively studied [15], [16]. For control, various linear and nonlinear control methods such as LQR, feedback linearized control, sliding model control, adaptive control, to name a few [17], [14], [18]. Recently, Chung et al. suggested an exponentially stable tracking control law for the attitude and position dynamics of formation flying [16]. Note that the most prior studies assume that the reference orbits are circular or elliptic, and are not perturbed by any disturbances. However, as the altitude of the orbit gets lower, the effect of the perturbations becomes noticeable.

In this paper, we introduce a new cooperative control approach, based on active adaptation of tracking/diffusive coupling gains and a distance-based connectivity condition. This strengthens results in [19]. In [19], the authors developed a new cooperative control, which is inspired by the knowledge feedback control [20]. The method actively tries to find tracking and diffusive coupling gains so that the formation system can be synchronized effectively in the sense of fuel consumption and time of convergence. In this study, we generalize this approach to network structures that are directed and unbalanced, i.e. where the number of inputs for coupling is not necessarily the same as that of outputs for any given spacecraft. We also introduce a connectivity 
condition, where the gains reduce as the distance between spacecraft increases. This concept is similar to the CuckerSmale method [21], which is a synchronization method based on the relative distance between agents in formation. Moreover, the method has been extended to spacecraft formation flying [22]. However the approach places specific conditions for synchronization while the proposed synchronization law provides more flexible topology without any requirements. It should be noted that the proposed adaptive synchronization law with the connectivity will eliminate the need for a balanced graph topology and can derive a stabilizing synchronization Laplacian on an unbalanced communication topology.

The organization of this paper is as follows: In Section II, the nonlinear dynamics of the reference and relative orbital motions are derived. In Section III, our mathematical tools are briefly introduced. The proposed cooperative control law is suggested in Section IV with stability analysis. In Section V, numerical validation is presented to compare the results with other control methods. Finally, in Section VI, concluding remarks are stated.

\section{Derivation of Exact Dynamics}

In this section, the exact nonlinear dynamic models for the reference orbit and the relative orbit are derived. The coordinates are shown in Fig. 1. For disturbance sources, the second zonal harmonic effect $\left(J_{2}\right)$ and atmospheric drag are taken into account, which are the major disturbances in LEO. Prior to the derivation of the dynamics, some disturbance models are needed. First, the gradient of the gravitational potential with the $J_{2}$ perturbation (in Local Vertical Local Horizontal (LVLH) frame) is written as [8]:

$\nabla U=\frac{\mu}{r^{2}} \hat{r}+\frac{k_{J_{2}} a^{2}}{r^{4}}\left(1-3 s_{i}^{2} s_{\theta}^{2}\right) \hat{r}+\frac{k_{J_{2}} a^{2} s_{i}^{2} s_{2 \theta}}{r^{4}} \hat{s}+\frac{k_{J_{2}} a^{2} s_{2 i} s_{\theta}}{r^{4}} \hat{w}$

where $\mu, a, i$, and $\theta$ denote gravitational parameter, semimajor axis, inclination, and argument of latitude, respectively. Moreover, $k_{J_{2}}=\frac{3}{2} J_{2} \mu R_{e}^{2}$, where $R_{e}$ is the radius of the Earth. Abbreviations $s_{(\cdot)}, c_{(\cdot)}$ stand for $\sin (\cdot), \cos (\cdot)$, respectively.

The effect of the atmospheric drag can be described by the corresponding acceleration (in a frame relative to rotating atmosphere) as [23]:

$$
\mathbf{a}_{\mathrm{drag}}=-\frac{1}{2} \frac{C_{D} A}{m} \rho v_{\mathrm{r}} \mathbf{V}_{\mathrm{r}}=:-C_{\mathrm{dn}} v_{\mathrm{r}} \mathbf{V}_{\mathrm{r}}
$$

where $C_{D}$ is coefficient of drag, $A$ is the cross-sectional area, $v_{\mathrm{r}}:=\left\|\mathbf{V}_{\mathrm{r}}\right\|$, and $\rho$ is the air density, which can be described as $\rho=\rho_{o} e^{\left[h_{o}-h\right] / H}$ where $h_{0}, h$, and $H$ are the reference altitude, the actual altitude, and the scale height, respectively. Note that $\mathbf{V}_{\mathrm{r}}$ is a velocity of the reference orbit relative to the rotating atmosphere, described in Earth Centered Inertial (ECI) frame, defined by

$$
\mathbf{V}_{\mathrm{r}}=\dot{\mathbf{r}}_{\mathrm{ECI}}-\boldsymbol{\omega}_{\mathrm{E}} \times \mathbf{r}_{\mathrm{ECI}}=\left[\begin{array}{lll}
\dot{X}+\omega_{e} Y & \dot{Y}-\omega_{e} X & \dot{Z}
\end{array}\right]^{T}
$$

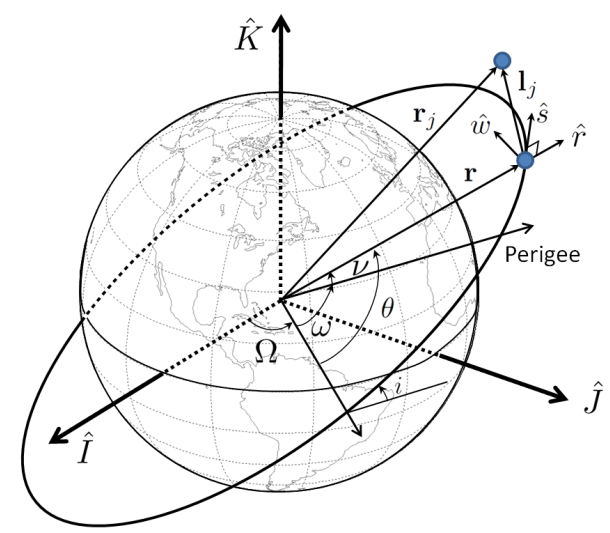

Fig. 1. Coordinates: ECI $(\hat{I}, \hat{J}, \hat{K})$ and $\operatorname{LVLH}(\hat{r}, \hat{s}, \hat{w})$

where $\boldsymbol{\omega}_{\mathrm{E}}=\left[\begin{array}{lll}0 & 0 & \omega_{e}\end{array}\right]^{T}$ and $\mathbf{r}_{\mathrm{ECI}}=\left[\begin{array}{lll}X & Y & Z\end{array}\right]^{T}$ denote the rate of rotation of the Earth and a position of spacecraft in ECI frame, respectively.

Because ECI and LVLH are different frames, a rotation matrix is needed. For convenience, the 3-1-3 Euler rotation matrix $\left(\mathbf{R}(\theta, i, \Omega):=\mathrm{ROT}_{3}(\theta) \mathrm{ROT}_{1}(i) \mathrm{ROT}_{3}(\Omega)\right.$, [LVLH] $=\mathbf{R}[\mathrm{ECI}]$, where $\Omega$ is right ascension of ascending node.) is used in this paper.

$$
\mathbf{R}=\left[\begin{array}{l}
R_{1} \\
R_{2} \\
R_{3}
\end{array}\right]:=\left[\begin{array}{ccc}
c_{\theta} c_{\Omega}-s_{\theta} c_{i} s_{\Omega} & c_{\theta} s_{\Omega}+s_{\theta} c_{i} c_{\Omega} & s_{\theta} s_{i} \\
-s_{\theta} c_{\Omega}-c_{\theta} c_{i} s_{\Omega} & -s_{\theta} s_{\Omega}+c_{\theta} c_{i} c_{\Omega} & c_{\theta} s_{i} \\
s_{i} s_{\Omega} & -s_{i} c_{\Omega} & c_{i}
\end{array}\right] .
$$

A. Nonlinear Dynamics for Reference Orbit under the $J_{2}$ Perturbation and Atmospheric Drag

The exact nonlinear dynamics for reference orbit with $J_{2}$ and atmospheric drag effects are obtained here.

Proposition 1: Consider the Earth which has nonspherical gravity due to the $J_{2}$ perturbation and atmospheric drag. Then the motion of spacecraft is governed by the following system of equations:

$$
\begin{aligned}
\frac{\mathrm{d} a}{\mathrm{~d} t}= & -\frac{2 a \chi \varepsilon}{\kappa^{2}}\left(e s_{\nu}\left(1-3 s_{i}^{2} s_{\theta}^{2}\right)+\chi s_{i}^{2} s_{2 \theta}\right) \\
& -\frac{2 a E}{\kappa^{2}}\left(e s_{\nu} R_{1}+\chi R_{2}\right) \mathbf{V}_{\mathrm{r}} \\
\frac{\mathrm{d} q_{1}}{\mathrm{~d} t}= & \varepsilon\left\{\left(\chi-(5 \chi+2) s_{i}^{2} s_{\theta}^{2}\right) c_{\theta}+2 e\left(-s_{\omega} c_{\theta}+s_{(\theta+\omega)}\right.\right. \\
& \left.\left.\times c_{i}^{2}\right) s_{\theta}\right\}+E\left\{\chi c_{\theta} R_{1}-\left((\chi+1) s_{\theta}+e s_{\omega}\right) R_{2}\right. \\
& \left.+\frac{e c_{\omega} c_{i} s_{\theta} R_{3}}{s_{i}}\right\} \mathbf{V}_{\mathrm{r}} \\
\frac{\mathrm{d} q_{2}}{\mathrm{~d} t}= & -\varepsilon s_{\theta}\left\{\chi\left(1-3 s_{i}^{2} s_{\theta}^{2}\right)+2(\chi+1) s_{i}^{2} c_{\theta}^{2}+2 e\left(c_{\omega} c_{\theta}\right.\right. \\
& \left.\left.-c_{(\theta+\omega)} c_{i}^{2}\right)\right\}-E\left\{\chi s_{\theta} R_{1}+\left((\chi+1) c_{\theta}+e c_{\omega}\right)\right. \\
& \left.\times R_{2}+\frac{e s_{\omega} c_{i} s_{\theta} R_{3}}{s_{i}}\right\} \mathbf{V}_{\mathrm{r}} \\
\frac{\mathrm{d} i}{\mathrm{~d} t}= & -\frac{\varepsilon s_{2 i} s_{2 \theta}}{2}-E c_{\theta} R_{3} \mathbf{V}_{\mathrm{r}} \\
\frac{\mathrm{d} \Omega}{\mathrm{d} t}= & -2 \varepsilon c_{i} s_{\theta}^{2}-\frac{E s_{\theta}}{s_{i}} R_{3} \mathbf{V}_{\mathrm{r}} \\
\frac{\mathrm{d} \theta}{\mathrm{d} t}= & \frac{n \chi^{2}}{\kappa^{3}}+2 \varepsilon c_{i}^{2} s_{\theta}^{2}+\frac{E c_{i} s_{\theta}}{s_{i}} R_{3} \mathbf{V}_{\mathrm{r}}
\end{aligned}
$$


where $e, \omega, \nu, n$ denote orbital elements: eccentricity, argument of periapsis, true anomaly, and mean motion, respectively. Moreover, $q_{1}$ and $q_{2}$ are defined as $q_{1}=$ $e s_{\omega}, q_{2}=e c_{\omega}$, respectively [24]. Note that $\kappa=\left(1-e^{2}\right)^{1 / 2}$, $\chi=1+e c_{\nu}, \varepsilon=k_{J_{2}} \chi^{3} / n a^{5} \kappa^{7}$, and $E=\kappa C_{\mathrm{dn}} v_{\mathrm{r}} / n a \chi$.

Proof: Given Gauss' variational equations [23], the system of equations for orbital elements with the $J_{2}$ perturbation can be obtained. Substituting the acceleration due to atmospheric drag (2) and the rotational matrix $\mathbf{R}$ into Gauss' variational equation, (3) can be derived. The detailed procedure is omitted.

One useful equation is $n:=\sqrt{\mu / a^{3}}$. The time derivative of the mean motion can be expressed as follows: $\dot{n}=$ $-3 n \dot{a} / 2 a$.

Remark 1: If the effect of atmospheric drag is omitted from (3), the equations describe the motion of spacecraft under the $J_{2}$ perturbation, which was derived in [8]. Equations for $\Omega, i, \theta(=\omega+\nu)$ can be obtained readily and the other equations also can be found by simple substitution. The reason to use the states in (3) is intuitive and easily obtained from position and velocity information while those in [8] need additional calculation because there is no direct transformation method from position and velocity.

Remark 2: The effect of the Earth rotation was considered in (3), which has not been taken into account in previous studies. Moreover, some prior studies used linearized atmospheric drag models and the velocity of the reference orbit. However, the velocity term should be relative to the air because the air on the Earth is not fixed to the Earth.

Remark 3: There is no singularity problem at $e=0$ or $i=0$ in (3). Note that there are $s_{i}$ in the denominators in (3). However, the first and second elements in $R_{3}$ have $s_{i}$. Moreover, $\dot{Z}=0$ at $i=0$. Therefore, the terms that contain $s_{i}$ in their denominators are bounded.

\section{B. Nonlinear Dynamics for Relative Orbits under the $J_{2}$ Perturbation and Atmospheric Drag}

The exact nonlinear relative dynamics under the second zonal harmonic effect and atmospheric drag is derived.

Proposition 2: The relative motion of spacecraft with respect to the reference trajectory given in (3) follows the equations as:

$$
\begin{aligned}
\ddot{x}_{j}= & -\dot{x}_{j} C_{\mathrm{dn}, j} v_{j \mathrm{r}}+2 \dot{y}_{j} \omega_{z}-x_{j}\left(\eta_{j}^{2}-\omega_{z}^{2}\right)+y_{j}\left(\alpha_{z}\right. \\
& \left.+C_{\mathrm{dn}, j} v_{j \mathrm{r}} \omega_{z}\right)-z_{j} \omega_{x} \omega_{z}-\left(\zeta_{j}-\zeta\right) s_{i} s_{\theta}-r\left(\eta_{j}^{2}-\eta^{2}\right) \\
& -C_{\mathrm{dn}, j} v_{j \mathrm{r}} R_{1} \mathbf{V}_{\mathrm{r}}+F_{j r} \\
\ddot{y}_{j}= & -2 \dot{x}_{j} \omega_{z}-\dot{y}_{j} C_{\mathrm{dn}, j} v_{j \mathrm{r}}+2 \dot{z}_{j} \omega_{x}-x_{j}\left(\alpha_{z}+C_{\mathrm{dn}, j} v_{j \mathrm{r}} \omega_{z}\right) \\
& -y_{j}\left(\eta_{j}^{2}-\omega_{z}^{2}-\omega_{x}^{2}\right)+z_{j}\left(\alpha_{x}-C_{\mathrm{dn}, j} v_{j \mathrm{r}} \omega_{x}\right)-\left(\zeta_{j}\right. \\
& -\zeta) s_{i} s_{\theta}-C_{\mathrm{dn}, j} v_{j \mathrm{r}} R_{2} \mathbf{V}_{\mathrm{r}}+F_{j s} \\
\ddot{z}_{j}= & -2 \dot{y}_{j} \omega_{x}-\dot{z}_{j} C_{\mathrm{dn}, j} v_{j \mathrm{r}}-x_{j} \omega_{x} \omega_{z}-y_{j}\left(\alpha_{x}+C_{\mathrm{dn}, j} v_{j \mathrm{r}}\right. \\
& \left.\times \omega_{x}\right)-z_{j}\left(\eta_{j}^{2}-\omega_{x}^{2}\right)-\left(\zeta_{j}-\zeta\right) c_{i}-C_{\mathrm{dn}, j} v_{j \mathrm{r}} R_{3} \mathbf{V}_{\mathrm{r}} \\
& +F_{j w}
\end{aligned}
$$

where $\eta_{j}, \eta$ describe angular velocities, $\zeta_{j}, \quad \zeta$ angular accelerations of the $j$ th and the chief spacecraft and $\alpha_{x}=$ $\dot{\omega}_{x}, \alpha_{z}=\dot{\omega}_{z}$ the elements of the time derivative of the angular velocity of the LVLH frame $\boldsymbol{\omega}_{\mathrm{I}}=\left[\begin{array}{lll}\omega_{x} & \omega_{y} & \omega_{z}\end{array}\right]^{T}$, respectively. Definitions of the variables can be found in [8]. Moreover, $\mathbf{F}_{j}:=\left[\begin{array}{lll}F_{j r} & F_{j s} & F_{j w}\end{array}\right]^{T}$ denotes a control input vector for the $j$ th spacecraft and $v_{j \mathrm{r}}=\left\|\mathbf{V}_{j \mathrm{r}}\right\|$, where $\mathbf{V}_{j \mathrm{r}}$ is the relative velocity of the $j$ th spacecraft with respect to the rotating Earth atmosphere.

Proof: The system of equations can be derived using the Lagrangian formulation $\frac{\mathrm{d}}{\mathrm{d} t}\left(\frac{\partial L_{j}}{\partial \dot{\mathbf{q}}_{j}}\right)-\frac{\partial L_{j}}{\partial \mathbf{q}_{j}}=\mathbf{F}_{j}$, where $L_{j}:=U_{\mathrm{K}, j}-U_{\mathrm{P}, j}$ denotes the Lagrangian, $U_{\mathrm{K}, j}$ the kinetic energy, and $U_{\mathrm{P}, j}$ the potential energy for the $j$ th spacecraft, respectively. The kinetic and potential energies of the $j$ th spacecraft can be derived easily. Note that since $\mathbf{V}_{j \mathrm{r}}$ is described in the LVLH frame, it can be expressed as:

$$
\begin{aligned}
\mathbf{V}_{j \mathrm{r}}= & \dot{\mathrm{i}}_{j}+\mathbf{R V}_{\mathrm{r}}=\left(\dot{x}_{j}-y_{j} \omega_{z}+R_{1} \mathbf{V}_{\mathrm{r}}\right) \hat{r}+\left(\dot{y}_{j}+x_{j} \omega_{z}\right. \\
& \left.-z_{j} \omega_{x}+R_{2} \mathbf{V}_{\mathrm{r}}\right) \hat{s}+\left(\dot{z}_{j}+y_{j} \omega_{x}+R_{3} \mathbf{V}_{\mathbf{r}} r\right) \hat{w}
\end{aligned}
$$

where $\mathbf{l}_{j}:=\left[\begin{array}{lll}x_{j} & y_{j} & z_{j}\end{array}\right]^{T}$ is the relative position of the $j$ th spacecraft in LVLH.

Substituting (2) for the $j$ th spacecraft and (5) into the Lagrangian formulation with $L_{j}$, the exact nonlinear relative motion with $J_{2}$ and atmospheric drag effects can be derived. Note that the exact nonlinear relative dynamic models including the $J_{2}$ perturbation can be found in [8]. The detailed procedure is omitted.

\section{Mathematical Tools and Stability Analysis}

Prior to developing a cooperative control law for spacecraft formation flying, necessary mathematical models are needed. We briefly review an important nonlinear stability tool and dynamic modeling of general spacecraft trajectory motion by using Lagrange's equation. Note that Lagrange's equation can also be applied to the spacecraft attitude dynamics. The equations of motion for spacecraft trajectories in LVLH frame with multiple degrees of freedom $\left(\mathbf{q}_{j} \in \mathbb{R}^{n}\right)$ can be written as:

$\mathbf{M}_{j}\left(\mathbf{q}_{j}\right) \ddot{\mathbf{q}}_{j}+\mathbf{C}_{j}(œ) \dot{\mathbf{q}}_{j}-\mathbf{G}_{j}\left(\mathbf{q}_{j}, œ\right)+\mathbf{D}_{j}\left(\mathbf{q}_{j}, \dot{\mathbf{q}}_{j}, œ\right)=\boldsymbol{\tau}_{j}$

where $j(1 \leq j \leq p)$ and $p$ denote the index of the dynamic systems in a formation and the total number of individual spacecraft, respectively. Here, $\mathbf{M}_{j}, \mathbf{G}_{j}$, and $\mathbf{D}_{j}$ describe the mass-inertia matrix, the force related to $J_{2}$ perturbation, and the force caused by the atmospheric drag, respectively. Parameters $\propto=\left[\begin{array}{llllll}a & e & i & \Omega & \omega & \nu\end{array}\right]^{T}$ and $\boldsymbol{\tau}_{j}$ describe the orbital elements obtained by (3), and a generalized force acting on the $j$ th system, respectively. Note that $\mathbf{C}_{j}\left(\mathbf{q}_{j}, \dot{\mathbf{q}}_{j}\right)$ is defined such that $\left(\dot{\mathbf{M}}_{j}-2 \mathbf{C}_{j}\right)$ is skew-symmetric [25], and this property plays a central role in the stability analysis using contraction theory [26]. Note that the Lagrangian dynamics in (6) is usually used for the dynamics for spacecraft attitude and translational motion. Based on the relative dynamic models in (4), $\mathbf{M}_{j}$ and $\mathbf{C}_{j}(œ)$ can be written as:

$$
\mathbf{M}_{j}=\left[\begin{array}{lll}
1 & 0 & 0 \\
0 & 1 & 0 \\
0 & 0 & 1
\end{array}\right], \quad \mathbf{C}_{j}=\left[\begin{array}{ccc}
0 & -2 \omega_{z} & 0 \\
2 \omega_{z} & 0 & -2 \omega_{x} \\
0 & 2 \omega_{x} & 0
\end{array}\right] \text {. }
$$


The vector $\mathbf{D}_{j}$ contains air drag terms and $\mathbf{G}_{j}$ can be found by the rest of the terms in (4).

We use the contraction analysis for stability analysis (see [26] for more details). The contraction analysis has more general and intuitive combination properties (e.g., hierarchies) than the passivity method and Lyapunov theory, since it involves a state-space rather than an input-output method. Moreover, a stronger form of stability can be proven, i.e., globally exponential or globally asymptotic stability (for a semi-contracting system).

Consider a deterministic and smooth nonlinear system

$$
\dot{\mathbf{x}}(t)=\mathbf{f}(\mathbf{x}(t), \mathbf{u}(\mathbf{x}, t), t)
$$

where $\mathbf{x}(t) \in \mathbb{R}^{n}$, and $\mathbf{f}: \mathbb{R}^{n} \times \mathbb{R}^{m} \times \mathbb{R}_{+} \rightarrow \mathbb{R}^{n}$. A virtual displacement, $\delta \mathbf{x}$ is defined as an infinitesimal displacement at fixed time.

Lemma 3: For the system in (7), if there exists a positive $\lambda$ and a uniformly positive definite metric, $\mathbf{M}(\mathbf{x}, t)=$ $\boldsymbol{\Theta}(\mathbf{x}, t)^{T} \boldsymbol{\Theta}(\mathbf{x}, t)$, where $\boldsymbol{\Theta}$ is some smooth coordinate transformation of the virtual displacement, $\delta \mathbf{z}=\boldsymbol{\Theta} \delta \mathbf{x}$, such that the associated generalized Jacobian, $\mathbf{F}$ is expressed as

$$
\mathbf{F}=\left(\dot{\boldsymbol{\Theta}}(\mathbf{x}, t)+\boldsymbol{\Theta}(\mathbf{x}, t) \frac{\partial \mathbf{f}}{\partial \mathbf{x}}\right) \boldsymbol{\Theta}(\mathbf{x}, t)^{-1} \leq-\lambda \mathbf{I},
$$

then all system trajectories converge globally exponentially fast to a single trajectory regardless of the initial conditions, with a global exponential convergence rate of the largest eigenvalues of the symmetric part of $\mathbf{F}$.

Such a system is said to be contracting. The proof can be found in [26]. Equivalently, the system is contracting if $\exists \lambda>0$ such that

$$
\dot{\mathbf{M}}+\left(\frac{\partial \mathbf{f}}{\partial \mathbf{x}}\right)^{T} \mathbf{M}+\mathbf{M} \frac{\partial \mathbf{f}}{\partial \mathbf{x}} \leq-2 \lambda \mathbf{M}
$$

Note that (9) is useful for the stability proof of a Lagrangian system, since the inertia matrix $\mathbf{M}_{j}$ in (6) can be chosen as the metric $\mathbf{M}$ in (9).

In the next section, we derive a new synchronization control law for spacecraft formation flying.

\section{Nonlinear CoOperative Control For FORMATION FLYING UNDER DISTURBANCES}

In this section, we present a main adaptive synchronization control law for spacecraft formation flying. Here, the adaptation law updates tracking and diffusive coupling gains based on the formation shape. In this paper, we define the tracking control and the diffusive coupling gains as $c_{j j}$ and $c_{j k}(j \neq k)$, respectively. As shall be seen later, the gains are used in the form of $c_{j j} \mathbf{s}_{j}+c_{j k} \mathbf{s}_{k}$. It can be reformulated as $\left(c_{j j}+c_{j k}\right) \mathbf{s}_{j}+c_{j k}\left(\mathbf{s}_{k}-\mathbf{s}_{j}\right)$, which shows that the two diffusive couplings are the same. Note that changing the gains would modify the Laplacian matrix thereby affecting the stability of the formation. However, the proposed method permits the adaptability of the Laplacian matrix without sacrificing the stability of the cooperative control of the formation flying system, which we call the adaptive Laplacian matrix. This matrix has control gains as
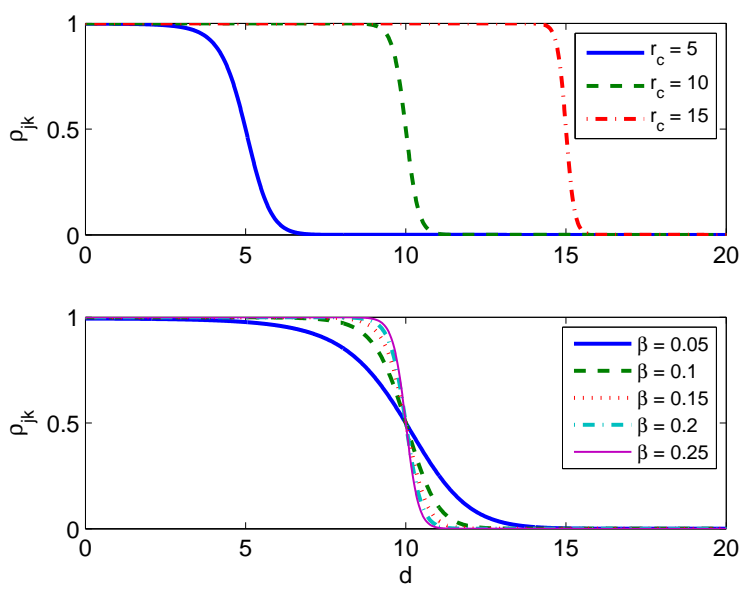

Fig. 2. Variation of connectivity with different $r_{c}(\beta=0.25$, upper figure $)$ and different $\beta\left(r_{c}=10\right.$, lower figure)

its elements and the gains are found by using the adaptive synchronization law. Using the adaptive Laplacian matrix, balanced graphs, which are important conditions in synchronization, can be relaxed for the formation, which is one of the main results in the current paper. In this paper, we further extend the cooperative control with the adaptation law to directed graphs (digraph) and unbalanced graphs. A new connectivity, based on relative distances among spacecraft, is introduced for effective synchronization. We start the design of the synchronization law with the connectivity method.

\section{A. Distance-Based Connectivity}

A new type of connectivity is introduced. The special connectivity, depending on the relative distance between the $j$ th and $k$ th spacecraft, is defined as

$$
\begin{aligned}
\text { if } d \leq d_{\text {limit }} & \varrho_{j k}(d)=\left(1+e^{\beta\left(d^{2}-r_{c}^{2}\right)}\right)^{-1} \\
\text { otherwise } & \varrho_{j k}(d)=0
\end{aligned}
$$

where $d:=\left\|\mathbf{r}_{j}-\mathbf{r}_{k}\right\|, r_{c}$ is a critical boundary, and $\beta$ determines an inclination of $\varrho_{j k}$ at $d=r_{c}$, respectively. In addition, $d_{\text {limit }}$ is defined by some small $\varrho_{\text {limit }}:=\varrho_{j k}\left(d_{\text {limit }}\right)>$ 0 such that $\varrho_{j k}(d)<\varrho_{\text {limit }}$ then $\varrho_{j k}(d)=0 \quad \forall d>d_{\text {limit }}$. The effects of the $r_{c}$ and $\beta$ can be seen in Fig. 2. In essence, by changing the two variables, we can control the number of connections among spacecraft in formation.

The connectivity has special characteristics as follow:

- $\varrho_{j k} \approx$ constant around the center and slowly decreases as $d$ approaches $r_{c}$ from $d=0$.

- $\varrho_{j k}=0.5$ at $d=r_{c}$

- $\varrho_{j k}$ decreases fast near $d=r_{c}$ depending on $\beta$ and has lower values as $d\left(>r_{c}\right)$ increases.

The connectivity shows that the any spacecraft can communicate each other and consider the other spacecraft motion if the relative distance between them is less than $d_{\text {limit }}$. Otherwise, the connectivity becomes zero as $d$ goes to infinity. If the connectivity is applied to spacecraft communication with adaptive control gains, we can expect flexible range of boundary for connection and intensity of the control gains 


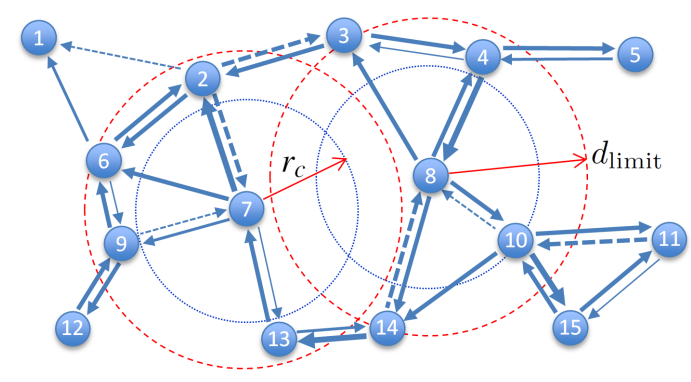

(a) Communication and diffusive couplings in formation by the proposed cooperative control

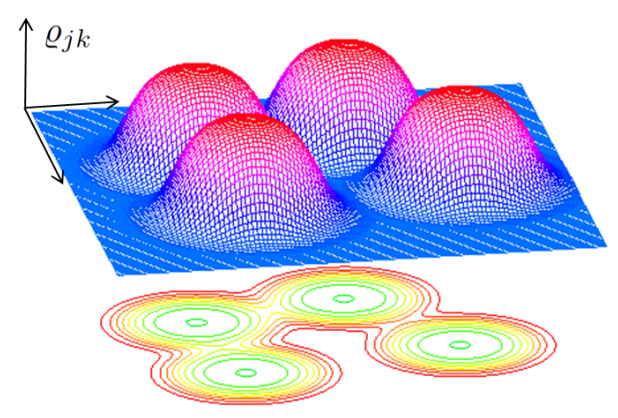

(b) connectivity for agents 2-3-8-7 in the upper figure

Fig. 3. Effects of the connectivity in (10). Only when spacecraft are located within $d_{\text {limit }}$, they can consider the diffusive coupling between them.

for the synchronization as in Fig. 3(a). That is, all spacecraft communicate with adjacent spacecraft within a range $\left(d_{\text {limit }}\right.$, the dashed circles). The dotted circles with $r_{c}$ denotes the critical boundary, which means that the connectivity becomes weak if $d>r_{c}$ and zero if $d>d_{\text {limit }}$. All communications are assumed to be directed with coupling gains. The different thicknesses of the arrows show the different coupling gains. The dashed arrows denotes negative values of $c_{j k}$. These phenomena for $c_{j k}$ can be obtained by the proposed adaptive cooperative control. Fig. 3(b) shows how spacecraft in formation can be connected by using the connectivity method.

Remark 4: This is similar to the Cucker-Smale method for synchronization [21] in the sense that they are distancebased method. However, as shall be seen in the main control law, the proposed connectivity does not affect the stability condition of the proposed synchronization law. Moreover, connection between spacecraft can be more flexible with different $\beta$ and $r_{c}$.

\section{B. Active Robust Adaptive Synchronization Control Law}

The active parameter adaptation is introduced. It is applied to tuning the tracking control gains and diffusive coupling gains. For this purpose, the proposed synchronization law can be written as:

$$
\begin{aligned}
\boldsymbol{\tau}_{j}= & \hat{\mathbf{M}}_{j} \ddot{\mathbf{q}}_{j, r}+\hat{\mathbf{C}}_{j}(\boldsymbol{œ}) \dot{\mathbf{q}}_{j, r}+\hat{\mathbf{D}}_{j}\left(\mathbf{q}_{j, r}, \dot{\mathbf{q}}_{j, r}, \boldsymbol{\mathbf { e }}\right)-k_{j} \mathbf{s}_{j} \\
& -\mathbf{W}_{j}\left(\mathbf{s}, \varrho_{j}\right) \mathbf{c}_{j} \\
= & \mathbf{Y}_{j}\left(\mathbf{q}_{j, r}, \dot{\mathbf{q}}_{j, r}, \ddot{\mathbf{q}}_{j, r}, \boldsymbol{e}\right) \hat{\mathbf{b}}_{j}-k_{j} \mathbf{s}_{j}-\mathbf{W}_{j}\left(\mathbf{s}, \varrho_{j}\right) \mathbf{c}_{j}
\end{aligned}
$$

where $\mathbf{s}_{j}:=\dot{\mathbf{q}}_{j}-\dot{\mathbf{q}}_{j, r}=\dot{\mathbf{q}}_{j}-\dot{\mathbf{q}}_{d}+\boldsymbol{\Lambda}\left(\mathbf{q}_{j}-\mathbf{q}_{d}\right)$ is a composite variable, $\mathbf{q}_{d}(t)$ a time-varying desired trajectory, $\boldsymbol{\Lambda}$ an $l \times l$ positive diagonal matrix, and $\varrho_{j}=\left[\begin{array}{llll}\varrho_{j 1} & \varrho_{j 2} & \cdots & \varrho_{j p}\end{array}\right]^{T}$, respectively. Moreover, the $l \times 1$ vector $\hat{\mathbf{b}}_{j}$ has $l$ uncertain parameters related to atmospheric drag in the dynamic models for its elements. The $n \times l$ matrix $\mathbf{Y}_{j}$ can be found from $\mathbf{Y}_{j} \mathbf{b}_{j}:=\mathbf{M}_{j} \ddot{\mathbf{q}}_{j, r}+\mathbf{C}_{j} \dot{\mathbf{q}}_{j, r}+\mathbf{D}_{j}$, where $\mathbf{b}_{j}$ is the real value of $\hat{\mathbf{b}}_{j}$. In this paper, all parameters related to the atmospheric drag are assumed to be unknown with boundaries in the $j$ th spacecraft $(1 \leq j \leq p)$. A positive constant $k_{j}$ is a tracking control gain for the $j$ th spacecraft. The $n \times p$ matrix $\mathbf{W}_{j}$ and the $p \times 1$ vector $\mathbf{c}_{j}$ are defined as $\mathbf{W}_{j}:=\left[\begin{array}{lllllll}\varrho_{j 1} \mathbf{s}_{1} & \cdots & \varrho_{j j-1} \mathbf{s}_{j-1} & \mathbf{s}_{j} & \varrho_{j j+1} \mathbf{s}_{j+1} & \cdots & \varrho_{j p} \mathbf{s}_{p}\end{array}\right]$ and $\mathbf{c}_{j}:=\left[\begin{array}{lllll}c_{j 1} & \cdots & c_{j 2} & \cdots & c_{j p}\end{array}\right]^{T}$, respectively. Note that $\mathbf{c}_{j}$ is a vector whose elements are tracking control and diffusive coupling gains for the $j$ th spacecraft, updated by the adaptation law described below. In this control law, $\mathbf{G}_{j}$ is not considered because most terms in $\mathbf{G}_{j}$ are differential $J_{2}$ perturbations, which imply that the magnitude of $\mathbf{G}_{j}$ is relatively smaller than other terms. Hence, $\mathbf{G}_{j}$ is regarded as a disturbance in this paper.

We introduce the adaptation law for the synchronization. Note that the variables in parentheses are omitted for simplicity from here. The individual dynamics of the formation are adaptive in the sense that their tracking control and diffusive coupling gains, denoted by $\mathbf{c}_{j}$, now adapt according to the adaptation law

$$
\dot{\mathbf{c}}_{j}=\boldsymbol{\Sigma}_{j} \mathbf{W}_{j}^{T} \mathbf{s}_{j}-\boldsymbol{\Sigma}_{j} \mathbf{S}_{\mathbf{c}_{j}} \mathbf{c}_{j}
$$

where the $p \times p$ matrix $\boldsymbol{\Sigma}_{j}=\operatorname{diag}\left(\sigma_{j 1}, \sigma_{j 2}, \cdots, \sigma_{j p}\right)$ is a positive diagonal matrix for the $j$ th spacecraft. The $p \times p$ diagonal matrix $\mathbf{S}_{\mathbf{c}_{j}}=\operatorname{diag}\left(\ell_{1} \ell_{2} \cdots \ell_{k} \cdots \ell_{p}\right)$ has elements $\ell_{k}(1 \leq k \leq p)$ with conditions: $\ell_{k}=$ $\left(\left|c_{j, k}\right|-c_{k, \max }\right) / c_{k, \max }$ if $\left|c_{j, k}\right|>c_{j, k \max }$, otherwise $\ell_{k}=0$, where $c_{k, \max }$ is defined as a component of $\mathbf{c}_{\max }$, representing a maximum or boundary value of $k$ th component $\left|c_{j, k}\right|$. These conditions are needed for the boundedness of $\hat{\mathbf{b}}_{j}$ [25]. It should be noted that $\mathbf{W}_{j}$ includes the connectivity, defined in (10). For instance, if the relative distance between the $j$ th and $k$ th spacecraft is greater than $d_{\text {limt }}$, the $k$ th element in $\mathbf{W}_{j}$ has smaller values, which implies that the relative information will be considered less for the adaptation of the control gains.

In addition to the adaptation of the synchronization gains, a different type of adaptive control is needed to deal with the parameter uncertainties in (6). For this work, the estimation law is defined as

$$
\dot{\hat{\mathbf{b}}}_{j}=-\boldsymbol{\Gamma}_{j} \mathbf{Y}_{j}^{T} \mathbf{s}_{j}-\boldsymbol{\Gamma}_{j} \mathbf{S}_{\hat{\mathbf{b}}_{j}}\left(\hat{\mathbf{b}}_{j}+\operatorname{sgn}\left(\hat{\mathbf{b}}_{j}\right) \mathbf{b}_{\max }\right)
$$

where $\boldsymbol{\Gamma}_{j}=\operatorname{diag}\left(\gamma_{j 1}, \gamma_{j 2}, \cdots, \gamma_{j l}\right)$ is a positive diagonal matrix for the $j$ th spacecraft. The $l \times l$ diagonal matrix $\mathbf{S}_{\hat{\mathbf{b}}_{j}}$ consists of elements $\varsigma_{i}(1 \leq i \leq l)$ with conditions: $\varsigma_{i}=$ $0.5\left(\left|\hat{b}_{j, i}\right|-b_{i, \max }\right) / b_{i, \max }$ if $\left|\hat{b}_{j, i}\right|>b_{i, \max }$, otherwise $\varsigma_{i}=0$. In addition, $\mathbf{b}_{\max }$ is an $l \times 1$ vector whose component $b_{i, \max }$ is greater than the corresponding elements $\left|b_{j, i}\right|$ in $\mathbf{b}_{j}(1 \leq j \leq$ $p$ ). The vector will be used for boundaries of the parameters 
in $\mathbf{b}_{j}$ for the parameter estimation $\hat{\mathbf{b}}_{j}$. Moreover, $\operatorname{sgn}\left(\hat{\mathbf{b}}_{j}\right)$ is an $l \times l$ diagonal matrix whose elements are sign functions [25] with respect to the corresponding elements in $\hat{\mathbf{b}}_{j}$.

We want to show the stability condition of the proposed synchronization law.

Theorem 4: The adaptive synchronization law in (11) globally asymptotically synchronizes the states of multiple adaptive dynamics in formation in the presence of disturbances.

Proof: Suppose that there are $p$ spacecraft in formation. Then from (6) and (11), the closed-loop dynamics for the $j$ th spacecraft can be expressed as

$$
\mathbf{M}_{j} \dot{\mathbf{s}}_{j}+\mathbf{C}_{j} \mathbf{s}_{j}+k_{j} \mathbf{s}_{j}-\mathbf{Y}_{j} \tilde{\mathbf{b}}_{j}+\mathbf{W}_{j} \mathbf{c}_{j}=\mathbf{G}_{j}
$$

where the variables in parentheses were omitted for simplicity. Note that $\mathbf{G}_{j}$ is regarded as non-vanishing disturbance and $\tilde{\mathbf{b}}_{j}:=\hat{\mathbf{b}}_{j}-\mathbf{b}_{j}$ and $\mathbf{b}_{j}$ is assumed to be constant or slowly varying. Therefore, $\dot{\tilde{\mathbf{b}}}_{j}=\dot{\hat{\mathbf{b}}}_{j}-\dot{\mathbf{b}}_{j}=\dot{\hat{\mathbf{b}}}_{j}$.

This system follows the adaptation law described in (12). Therefore, the closed-loop system for the formation, the parameter adaptation law, and the parameter estimation comprised of $p$ adaptive systems can be written as

$$
\begin{aligned}
& {[\mathbf{M}] \dot{\mathbf{x}}+[\mathbf{C}] \mathbf{x}+[\mathbf{K}] \mathbf{x}+[\mathbf{W}]\{\mathbf{c}\}-[\mathbf{Y}]\{\tilde{\mathbf{b}}\}=\{\mathbf{G}\}} \\
& \{\dot{\mathbf{c}}\}=[\boldsymbol{\Sigma}][\mathbf{W}]^{T} \mathbf{x}-[\boldsymbol{\Sigma}]\left[\mathbf{S}_{\mathbf{c}}\right]\{\mathbf{c}\} \\
& \{\dot{\tilde{\mathbf{b}}}\}=-[\boldsymbol{\Gamma}][\mathbf{Y}]^{T} \mathbf{x}-[\boldsymbol{\Gamma}]\left[\mathbf{S}_{\hat{\mathbf{b}}}\right]\left(\{\hat{\mathbf{b}}\}+[\operatorname{sgn}(\{\hat{\mathbf{b}}\})]\left\{\mathbf{b}_{\max }\right\}\right)
\end{aligned}
$$

or, in a matrix form as

$$
\begin{aligned}
& {\left[\begin{array}{ccc}
{[\mathbf{M}]} & \mathbf{0} & \mathbf{0} \\
\mathbf{0} & {\left[\boldsymbol{\Sigma}^{-1}\right]} & \mathbf{0} \\
\mathbf{0} & \mathbf{0} & {\left[\boldsymbol{\Gamma}^{-1}\right]}
\end{array}\right]\left[\begin{array}{c}
\dot{\mathbf{x}} \\
\{\dot{\mathbf{c}}\} \\
\tilde{\tilde{\mathbf{b}}}\}
\end{array}\right]+\left[\begin{array}{ccc}
{[\mathbf{C}]} & \mathbf{0} & \mathbf{0} \\
\mathbf{0} & \mathbf{0} & \mathbf{0} \\
\mathbf{0} & \mathbf{0} & \mathbf{0}
\end{array}\right]\left[\begin{array}{c}
\mathbf{x} \\
\{\mathbf{c}\} \\
\{\tilde{\mathbf{b}}\}
\end{array}\right]} \\
& +\left[\begin{array}{ccc}
{[\mathbf{K}]} & {[\mathbf{W}]} & -[\mathbf{Y}] \\
-[\mathbf{W}]^{T} & {\left[\mathbf{S}_{\mathbf{c}}\right]} & \mathbf{0} \\
{[\mathbf{Y}]^{T}} & \mathbf{0} & {\left[\mathbf{S}_{\hat{\mathbf{b}}}\right]}
\end{array}\right]\left[\begin{array}{c}
\mathbf{x} \\
\{\mathbf{c}\} \\
\{\tilde{\mathbf{b}}\}
\end{array}\right]=\left[\begin{array}{c}
\{\mathbf{G}\} \\
\mathbf{0} \\
-\left[\mathbf{S}_{\hat{\mathbf{b}}}\right]\left\{\mathbf{b}_{r}\right\}
\end{array}\right]
\end{aligned}
$$

where $[\mathbf{M}]=\operatorname{diag}\left(\mathbf{M}_{1}, \quad \mathbf{M}_{2}, \quad \cdots, \quad \mathbf{M}_{p}\right)$, $[\mathbf{C}]=\operatorname{diag}\left(\mathbf{C}_{1}, \quad \mathbf{C}_{2}, \quad \cdots, \quad \mathbf{C}_{p}\right), \quad \mathbf{x}=$ $\left[\begin{array}{llll}\mathbf{s}_{1}^{T}, & \mathbf{s}_{2}^{T} & \cdots & \mathbf{s}_{p}^{T}\end{array}\right]^{T},\left[\boldsymbol{\Sigma}^{-1}\right]=\operatorname{diag}\left(\boldsymbol{\Sigma}_{1}^{-1}, \boldsymbol{\Sigma}_{2}^{-1}, \cdots, \boldsymbol{\Sigma}_{p}^{-1}\right)$, $\left[\boldsymbol{\Gamma}^{-1}\right]=\operatorname{diag}\left(\boldsymbol{\Gamma}_{1}^{-1}, \quad \boldsymbol{\Gamma}_{2}^{-1}, \cdots, \boldsymbol{\Gamma}_{p}^{-1}\right)$, and $[\mathbf{K}]=$ $\operatorname{diag}\left(k_{1} \mathbf{I}_{p} \quad k_{2} \mathbf{I}_{p} \quad \cdots \quad k_{p} \mathbf{I}_{p}\right)$ where $\mathbf{I}_{p}$ is a $p \times p$ identity matrix, respectively. The vector $\left\{\mathbf{b}_{r}\right\}$ is defined as $\left\{\mathbf{b}_{r}\right\}=\{\mathbf{b}\}+[\operatorname{sgn}(\{\hat{\mathbf{b}}\})]\left\{\mathbf{b}_{\max }\right\}$.

Because of the non-vanishing disturbance $\{\mathbf{G}\}$, the adapted control gains, and the estimated parameters in the dynamics, we prove the stability under the condition of the convergence of the control gains and the parameters. First, suppose that the control gains and the parameters are outside the boundaries at time $t_{0}$, i.e., $\left|\hat{b}_{j, i}\right|>b_{i, \max }$ and $\left|c_{j, k}\right|>c_{k, \text { max }}$ $(1 \leq j, k \leq p, 1 \leq i \leq l)$. Then we define a squared virtual length to prove the stability by using the contraction analysis as

$$
V_{\text {out }}:=\left[\begin{array}{c}
\delta \mathbf{x} \\
\delta\{\mathbf{c}\} \\
\delta\{\tilde{\mathbf{b}}\}
\end{array}\right]^{T}\left[\begin{array}{ccc}
{[\mathbf{M}]} & \mathbf{0} & \mathbf{0} \\
\mathbf{0} & {\left[\boldsymbol{\Sigma}^{-1}\right]} & \mathbf{0} \\
\mathbf{0} & \mathbf{0} & {\left[\boldsymbol{\Gamma}^{-1}\right]}
\end{array}\right]\left[\begin{array}{c}
\delta \mathbf{x} \\
\delta\{\mathbf{c}\} \\
\delta\{\tilde{\mathbf{b}}\}
\end{array}\right] .
$$

Differentiating (15) with respect to time with $\{\mathbf{G}\}=\mathbf{0}$, the virtual length analysis shows the condition as below:

$$
\begin{aligned}
\dot{V}_{\text {out }}= & -2\left[\begin{array}{c}
\delta \mathbf{x} \\
\delta\{\mathbf{c}\} \\
\delta\{\tilde{\mathbf{b}}\}
\end{array}\right]^{T}\left[\begin{array}{ccc}
{[\mathbf{K}]} & \mathbf{0} & \mathbf{0} \\
\mathbf{0} & {\left[\mathbf{S}_{\mathbf{c}}\right]} & \mathbf{0} \\
\mathbf{0} & \mathbf{0} & {\left[\mathbf{S}_{\hat{\mathbf{b}}}\right]}
\end{array}\right]\left[\begin{array}{c}
\delta \mathbf{x} \\
\delta\{\mathbf{c}\} \\
\delta\{\tilde{\mathbf{b}}\}
\end{array}\right] \\
& -2 \delta\{\tilde{\mathbf{b}}\}^{T}\left[\mathbf{S}_{\hat{\mathbf{b}}}\right] \delta\left\{\mathbf{b}_{r}\right\} .
\end{aligned}
$$

Note that the last term in the right hand side is positive for all $\{\hat{\mathbf{b}}\}$ because of the relationship among $\hat{\mathbf{b}}_{j}, \mathbf{b}_{j}$, and $\mathbf{b}_{\max }$. Therefore, by the incremental input-to-state stability (ISS) [27] and the contraction analysis, (14) is contracting by the negative definite Jacobian. Hence, we conclude that the system is incrementally input-to-state stable as long as $\|\mathbf{x}\|>\|\{\mathbf{G}\}\|,\left|\hat{b}_{j, i}\right|>b_{i, \max }$, and $\left|c_{j, k}\right|>c_{k, \max }$ for all $i, j, k(1 \leq j, k \leq p, 1 \leq i \leq l)$.

Suppose that some components in the control gains or the parameters are inside the boundaries, i.e., $\left|\hat{b}_{j, i}\right|<b_{i, \text { max }}$ or $\left|c_{j, k}\right|<c_{k, \max }$ for some $i, k(1 \leq i \leq l, 1 \leq k \leq$ $p$ ). By the definition, $\mathbf{S}_{\hat{\mathbf{b}}_{j}, i}=0$ and $\mathbf{S}_{\mathbf{c}_{j}, k}=0$ if $\left|\hat{b}_{j, i}\right|<$ $b_{i, \text { max }}$ and $\left|c_{j, k}\right|<c_{k, \text { max }}$, respectively. Note that because of (12) and (13), $\{\hat{\mathbf{b}}\}$ and $\{\mathbf{c}\}$ converge to the some values inside their boundaries in finite time. Therefore without loss of generality, we suppose $\left|\hat{b}_{j, i}\right|<b_{i, \max }$ and $\left|c_{j, k}\right|<c_{k, \max }$ for all $i, j, k(1 \leq j, k \leq p, 1 \leq i \leq l)$. Then, a squared virtual length can be defined as

$$
V_{\text {in }}:=\delta \mathbf{x}^{T}[\mathbf{M}] \delta \mathbf{x} .
$$

Due to the boundedness of $\hat{\mathbf{b}}_{j}, \mathbf{c}_{j}, \mathbf{Y}_{j}$, and $\varrho_{j},[\mathbf{Y}]\{\tilde{\mathbf{b}}\}$ and $[\mathbf{W}]\{\mathbf{c}\}$ can be regarded as bounded disturbances like $\{\mathbf{G}\}$ for the condition above. The time derivative of the virtual length without $\{\mathbf{G}\},\{\hat{\mathbf{b}}\}$, and $\{\mathbf{c}\}$ shows that

$$
\dot{V}_{\text {in }}=-2 \delta \mathbf{x}^{T}[\mathbf{K}] \delta \mathbf{x}<0 .
$$

Therefore, the system is contracting with the negative definite Jacobian as long as $\|\mathbf{x}\|>\Delta(\{\mathbf{G}\},\{\tilde{\mathbf{b}}\},\{\mathbf{c}\})$ where $\Delta$ is defined as $\Delta:=\sup _{\{\mathbf{G}\},\{\tilde{\mathbf{b}}\},\{\mathbf{c}\}}\|\{\mathbf{G}\}+[\mathbf{Y}]\{\tilde{\mathbf{b}}\}-[\mathbf{W}]\{\mathbf{c}\}\|$, which is bounded. From the results above, we conclude that the system is incrementally input-to-state stable, which implies that the multiple system is globally asymptotically synchronized with $\|\mathbf{x}\|>\Delta$.

Remark 5: It is noted that the synchronization law in (11) suggests a flexible control design for complex formation systems since control designers do not need to consider the exact values of the coupling gains depending on a particular graph topology of the formation. The gains $\left(c_{j k}\right)$ are automatically calculated based on both the adaptation law (12), the synchronization, the tracking errors, and the connectivity condition.

Remark 6: In [19], the authors suggested a flexible topology design method. However, the current paper requires a relaxed condition: we eliminated the constraints that the topology should be a balanced graph. The topology can be further extended to any unbalanced graph structure based on the connectivity in (10). The proposed method designs the topology based on the state errors, adaptation laws, and 


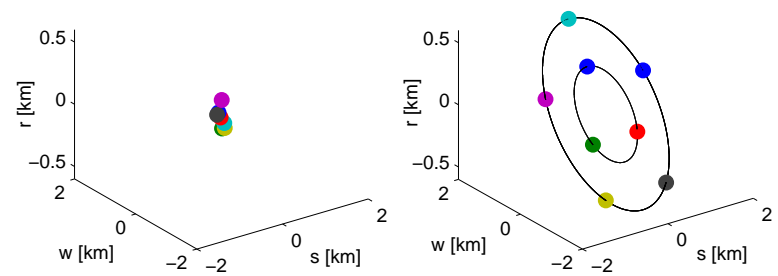

Fig. 4. Initial positions (left) and desired trajectories (right) of the spacecraft

hardware specification (i.e., possible number of connection, based on the connectivity condition). Note that the proposed synchronization law can be extended to heterogeneous systems.

\section{Numerical VALIDATION}

In this section, results of numerical simulation are presented to validate the proposed adaptive synchronization law. A desired trajectory, defined relative to the reference trajectory, is needed for simulation. We chose a periodic solution of the HCW equation [2]: $\dot{y}_{0}=-2 n x_{0}$ and $y_{0}=2 \dot{x}_{0} / n$. Note that a projected circular orbit with 26.565 deg between the cross-track and radial directions can be considered with additional conditions: $z_{0}= \pm 2 x_{0}$ and $\dot{z}_{0}= \pm \dot{x}_{0}$. Therefore, any projected circular orbit can be obtained with $x_{0}$ and $\dot{x}_{0}$. However, because of the linearization in HCW equation and disturbances, this condition does not hold as $x_{0}$ and $\dot{x}_{0}$ increase (i.e., the orbits will drift.). Therefore, proper controllers are needed for the formation keeping and reconfiguration.

For the simulation, the initial conditions for reference and relative orbits are needed: $a=6878.137 \mathrm{~km}, e=0$, $i=45 \mathrm{deg}, \Omega=30 \mathrm{deg}, \omega=0 \mathrm{deg}$, and $\nu=0 \mathrm{deg}$ for the initial reference orbit, $x_{0}=0.3,0.5 \mathrm{~km}$ and $\dot{x}_{0}=0 \mathrm{~km} / \mathrm{s}$ for the initial relative orbits (two periodic relative orbits), respectively. It is assumed that there are 8 spacecraft in formation: 3 spacecraft are located in the inner orbits and the other 5 spacecraft are in the outer orbits. Fig. 4 shows the initial position (left) and the desired trajectories (right) of the spacecraft in the formation. The mass for the spacecraft is $m=100 \mathrm{~kg}$ and the effective cross-sectional area of each spacecraft is $A=1 \mathrm{~m}^{2}$ (a sphere shape, i.e., $C_{D}=2.0$ [23]), respectively. For fixed coupling gains, $k_{j}=0.01$ was chosen for all spacecraft. All values for initial $c_{j k}$ were set to zeros.

The initial positions of the 8 spacecraft were randomly chosen by the Gaussian distribution with $\sigma=0.1 \mathrm{~km}$ for all three directions. The initial velocities of the spacecraft were assumed to be zero. The gain $\boldsymbol{\Sigma}_{j}$ for the adaptation law is set to $\sigma_{j j}=0.003$ and $\sigma_{j k}=0.001(j=1,2, \cdots, 8)$, respectively. For the estimation of the parameters, we will use composite variable $\hat{b}_{j}:=\hat{C}_{j}=\frac{\hat{C}_{D} \hat{\rho}_{j} \hat{A}_{j}}{2 \hat{m}_{j}}$.

\section{A. Performance of the Distance-Based Connectivity $\varrho$}

We evaluate the distance-based connectivity in (10). Note that it calculates the intensities $\left(\varrho_{j k}\right)$ based on the relative distances. For simulation, $\beta=20$ and $r_{c}=1.2 \mathrm{~km}$ for the

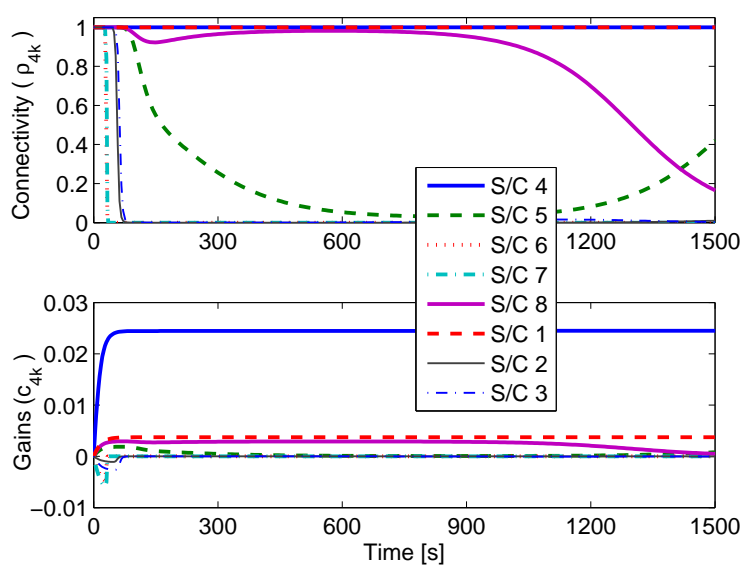

(a) Variation of the connectivity and the associated tracking control and diffusive coupling gains for the 4th spacecraft

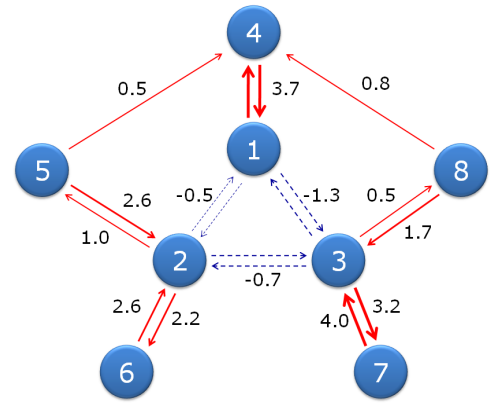

(b) Coupling status from the simulation

Fig. 5. Simulation results for the connectivity and the associated control gains, obtained by the proposed adaptive synchronization control law. In (b), the numbers indicate the diffusive coupling gains $\left(\times 10^{-3}\right)$.

inner orbits and $\beta=15$ and $r_{c}=1.5 \mathrm{~km}$ for the outer orbits were used. The upper figure in Fig. 5(a) shows the variations of the connectivity $\varrho_{j k}$ during $1500 \mathrm{sec}$. Since spacecraft were initially located in a small area (See Fig. 4.), each connectivity has a large value at the initial stage. However, because of the desired trajectories, positions of the spacecraft were expanded. At this time, the relative distances get increased, which affect the connectivity (30-70 sec). Finally, only some $\varrho_{j k}$ have values based on the final relative distances among the neighbors. Note that since the desired orbits are ellipses, the values of the connectivity can be changed.

The connectivity also influences the coupling gains as can be seen in the lower figure in Fig. 5(a): the coupling gains are obtained by $\varrho_{j k} c_{j k}$. That is, smaller $\varrho_{j k}$ makes less coupling gains (from the initial time to $50 \mathrm{sec}$ ). In other words, the communication gets weaker as the distance between spacecraft increases. Note that if there is no connectivity method, all $c_{j k}$ will have values regardless of relative distances, which is neither possible nor economical due to the communication load. On the other hand, if we use the 
connectivity, unnecessary long-distance communication can be eliminated.

\section{B. Performance of Proposed Adaptive Synchronization Law}

The proposed adaptive synchronization law in (11) includes the distance-based connectivity and the adaptation law. The adaptation law calculates tracking control and diffusive coupling gains based on the information of the state errors and connectivity. Therefore, not all spacecraft have to keep the same coupling gains for the synchronization. Fig. 5(b) shows the different control gains obtained from the simulation. It should be noted that some control gains have negative values, which implies that negative control gains can make the formation synchronized with fewer control inputs. Moreover, the overall connection in the formation is not balanced, while the system is well synchronized.

From the simulation, we could see how the connectivity method affects the communication/control gains for synchronization and the proposed adaptive synchronization law generates control inputs for synchronization. In addition, we could evaluate the performance of the synchronization law controlled by the proposed law. The robust stability of the proposed method due to the parameter uncertainties was also verified from the results of the simulation.

\section{CONCLUSIONS}

In this paper, we investigated a new cooperative control strategy for spacecraft formation flying. The exact nonlinear dynamic models of reference and relative orbital motions, which include $J_{2}$ and atmospheric drag effects as disturbances, were derived by using Gauss' variational equation and the Lagrangian formulation, respectively. The robust stability was proven by using the contraction analysis and the incremental input-to-state stability. The main contribution of the paper is the relaxation of the requirement of a network topology. That is, the proposed cooperative control law automatically computes suitable coupling gains based on the synchronization law, the state errors, and the relative distance between spacecraft in formation. Moreover, the connectivity based adaptation method exploits the relative distance information, thereby ensuring the stability of a complex network comprised of a large number of spacecraft. The proposed synchronization can be applied to more generalized robotic networks comprised of complex Lagrangian dynamics. By using results of the simulation, we demonstrated the effectiveness of the proposed synchronization method.

\section{ACKNOWLEDGMENTS}

Part of this research was carried out at the Jet Propulsion Laboratory, California Institute of Technology, under a contract with the National Aeronautics and Space Administration.

\section{REFERENCES}

[1] D. Scharf, F. Hadaegh, Z. Rahman, J. Shields, and G. Singh, "An Overview of the Formation and Attitude Control System for the Terrestrial Planet Finder Formation Flying Interferometer," in Int. Symp. Formation Flying Missions and Tech. Washington, D.C.: JPL, NASA, September 2004.
[2] W. Clohessy and R. Wiltshire, "Terminal Guidance System for Satellite Rendezvous," J. Astronaut. Sci., vol. 27, no. 9, pp. 653-658, 1960.

[3] J. Tschauner and P. Hempel, "Rendezvous zu einem in elliptischer Bahn umlaufenden Ziel," Acta Astronautica, vol. 11, no. 2, pp. 104 109, 1965.

[4] S. Schweighart and R. Sedwick, "High-Fidelity Linearized $J_{2}$ Model for Satellite Formation Flight," J. Guid., Contr., Dyn., vol. 25, no. 6, pp. 1073-1080, 2002.

[5] I. Ross, "Linearized Dynamic Equations for Spacecraft Subject to $J_{2}$ Perturbations," J. Guid., Contr., Dyn., vol. 26, no. 3, pp. 657-659, 2003

[6] D. Gim and K. Alfriend, "State Transition Matrix of Relative Motion for the Perturbed Noncircular Reference Orbit," J. Guid., Contr., Dyn., vol. 26, no. 6, pp. 956-971, 2003.

[7] J. Kechichian, "Motion in General Elliptic Orbit with Respect to a Dragging and Precessing Coordinate Frame," J. Astronaut. Sci., vol. 46, no. 1, pp. 25-45, 1998.

[8] G. Xu and D. Wang, "Nonlinear Dynamic Equations of Satellite Relative Motion Around an Oblate Earth," J. Guid., Contr., Dyn. vol. 31, no. 5, pp. 1521-1524, 2008.

[9] J. Shamma, Cooperative Control of Distributed Multi-Agent Systems. West Sussex, England: John Wiley \& Sons, Ltd, 2007.

[10] S.-J. Chung and J.-J. Slotine, "Cooperative Robot Control and Concurrent Synchronization of Lagrangian Systems," IEEE Trans. Robot., vol. 25, no. 3, pp. 686-700, 2009.

[11] D. Scharf, F. Hadaegh, and S. Ploen, "A Survey of Spacecraft Formation Flying Guidance and Control (Part I): Guidance," in Proc. Ameri. Contr. Conf., Denver, CO, June 2003, pp. 1733-1739.

[12] —, "A Survey of Spacecraft Formation Flying Guidance and Control (Part II): Control," in Proc. Ameri. Contr. Conf., Boston, MA, June 2004, pp. 2976-2984.

[13] R. Beard, J. Lawton, and F. Hadaegh, "A Coordination Architecture for Spacecraft Formation Control,” IEEE Trans. Contr. Syst. Technol., vol. 9, no. 6, pp. 777-790, 2001

[14] P. Wang, F. Hadaegh, and K. Lau, "Synchronized Formation Rotation and Attitude Control of Multi Free-Flying Spacecraft," J. Guid., Contr., Dyn., vol. 22, no. 1, pp. 28-35, 1999.

[15] W. Ren and R. Beard, "Decentralized Scheme for Spacecraft Formation Flying via the Virtual Structure Approach," J. Guid., Contr., Dyn., vol. 27, no. 1, pp. 73-82, 2004.

[16] S.-J. Chung, U. Ahsun, and J.-J. Slotine, "Application of Synchronization to Formation Flying Spacecraft: Lagrangian Approach,” J. Guid., Contr., Dyn., vol. 32, no. 2, 2009.

[17] G. Xing, S. Parver, and D. Folta, "Design and Implementation of Synchronized Autonomous Orbit and Attitude Control for Multiple Spacecraft Formation Using GPS Measurement Feedback," in AAS/AIAA Spaceflight Mech. Meeting, 2000, pp. 115-134.

[18] M. de Queiroz, V. Kapila, and Q. Yan, "Adaptive Nonlinear Control of Multiple Spacecraft Formation Flying," J. Guid., Contr., Dyn., vol. 23, no. 3, pp. 385-390, 2000.

[19] I. Chang and S.-J. Chung, "Bio-Inspired Adaptive Cooperative Control of Heterogeneous Robotic Networks," in AIAA Guid., Nav., Contr. Conf., Chicago, IL, August 2009, AIAA 2009-5886.

[20] W. Wang and J.-J. Slotine, "A Theoretical Study of Different Leader Roles in Networks," IEEE Trans. Automat. Contr., vol. 51, no. 7, pp. 1156-1161, 2006.

[21] F. Cucker and S. Smale, "Emergent Behavior in Flocks," IEEE Trans. Automat. Contr., vol. 52, no. 5, pp. 852-862, 2007.

[22] L. Perea, G. Gomez, and P. Elosegui, "Extension of the Cucker-Smale Control Law to Space Flight Formations," J. Guid., Contr., Dyn., vol. 32, no. 2, pp. 526-536, 2009.

[23] D. Vallado, Fundamentals of Astrodynamics and Applications, 3rd edition. Hawthorne, CA: Microcosm Press/Springer, 2007.

[24] H. Schaub and J. Junkins, Analytical Mechanics of Space Systems, AIAA Education Series. Reston, VA: AIAA, 2003.

[25] J.-J. Slotine and W. Li, Applied Nonlinear Control. Englewood Cliffs, NJ: Prentice Hall, 1991.

[26] W. Lohmiller and J.-J. Slotine, "On Contraction Analysis for NonLinear Systems," Automatica, vol. 34, no. 6, pp. 683-696, 1998.

[27] D. Angeli, "A Lyapunov Approach to Incremental Stability Properties," IEEE Trans. Automat. Contr., vol. 47, no. 3, pp. 410-421, 2002. 